\title{
Evaluation de quatre tests de diagnostic : frottis sanguins, CATT, IFI et ELISA-Ag dans l'étude de l'épidémiologie de la trypanosomose cameline à Trypanosoma evansi en Mauritanie
}

\author{
M.L. Dia ${ }^{1}$ N. Van Meirvenne ${ }^{2}$ E. Magnus ${ }^{2}$ \\ A.G. Luckins ${ }^{3}$ C. Diop ${ }^{1}$ A. Thiam ${ }^{1}$ \\ P. Jacquiet ${ }^{4}$ R. Hamers ${ }^{5}$
}

\section{Mots-clés}

Dromadaire - Trypanosoma evansi Réaction d'agglutination - Immunofluorescence - Test ELISA - Région agroclimatique - Conduite du troupeau Epidémiologie - M auritanie.

\begin{abstract}
Résumé
Une enquête sur l'épidémiologie de la trypanosomose cameline à T. evansi en Mauritanie a été conduite sur 2078 dromadaires de tous âges dans quatre régions (Trarza, Gorgol, Adrar, Hodh El Chargui) à caractéristiques climatiques et écologiques différentes. La prévalence de l'infection a été déterminée par l'examen de frottis sanguins et par trois tests sérologiques, le card agglutination test for trypanosomosis (CATT), I'immunofluorescence indirecte (IFI) pour la détection d'anticorps et l'enzyme-linked immunosorbent assay (ELISA) pour la détection d'antigènes. La prévalence globale de I'infection était de 1,4 p. 100 à l'examen parasitologique. La séroprévalence était de 16,5 p. 100 au CATT, de 24,3 p. 100 à I'IFI et de 14, 1 p. 100 à I'ELISA-Ag. La prévalence variait selon la région, la stratégie de conduite d'élevage pratiquée par les éleveurs, les troupeaux et l'âge des animaux. Cette enquête a montré que la trypanosomose cameline était présente en Mauritanie, surtout dans les zones boisées, près des cours d'eau fréquentés par les animaux.
\end{abstract}

- INTRODUCTION

La Mauritanie est un pays quasi désertique où les conditions physiques, climatiques et socioculturelles sont particulièrement favorables à l'élevage des dromadaires. On estime son cheptel à 1050000 dromadaires (8).

1. CNERV, BP 167, Nouakchott, Mauritanie

2. Institut de Médecine Tropicale Prince Léopold, Nationalestraat 155, B-2000 Anvers, Belgique

3. CTVM, Easter Bush, Midlothian, Edinburgh EH25 9RG, United Kingdom

4. Laboratoire de parasitologie, Ecole nationale vétérinaire de Toulouse, 23, chemin des Capelles, 31076, Toulouse, France

5. Instituut Voor Moleculaire Biologie, VUB Paardenstraat 65, B-1640 Saint-Genesius Rode, Belgium
La trypanosomose à Trypanosoma evansi est considérée comme une pathologie dominante des dromadaires (14). Elle est appelée localement « Tabourit » par les éleveurs mauritaniens qui l'associent, à tort ou à raison, avec certaines manifestations cliniques. Pourtant, les symptômes de la trypanosomose cameline ne sont pas pathognomoniques. Or, pour maîtriser une infection, un diagnostic sensible et spécifique est nécessaire. Observer T. evansi dans le sang périphérique des animaux étant fastidieux, de nombreux tests ont été mis au point pour combler les lacunes de l'examen du frottis sanguin dans le diagnostic de cette maladie $(1,2$, $11,12,13,16,19,22,23,26)$. Ces tests ont toutefois une spécificité, une sensibilité et une utilisation pratique sur le terrain différentes les unes des autres (20).

Dans la présente étude, quatre tests (examen de frottis sanguins, CATT, IFI et ELISA-Ag) ont été utilisés dans l'étude de l'épidémiologie de la trypanosomose cameline en Mauritanie. 


\section{MATERIEL ET METHODES}

\section{Zones d'enquêtes}

L'étude couvrait quatre régions (figure 1), de climat et d'écologie différents ou l'élevage des camelins est de type traditionnel et extensif.

\section{Trarza}

Cette région, à vocation pastorale, peut être divisée en deux zones : une zone sud avec des collections d'eau permanentes (fleuve Sénégal, Lac R'Kiz) ou temporaires, et une zone nord, sont de $20,4^{\circ} \mathrm{C}$ et $36,1^{\circ} \mathrm{C}$ et l'humidité relative minimale et maximale moyenne est de 24 p. 100 et 71 p. 100 . La végétation est composée d'un tapis herbacé (Cenchrus biflorus, Aristida et Eragrostis spp., etc.) et de ligneux (Acacia senegal, Balanites aegyptiaca, etc.).

Deux modes d'élevage camelin y sont pratiqués : l'élevage qui reste dans le sud de la région ou qui y descend en période de soudure (stratégie sud) et celui qui est confiné au nord toute l'année pour fuir les zones à « mouches » (stratégie nord).

\section{Gorgol}

C'est une région à vocation agricole. La pluviométrie annuelle est supérieure à $200 \mathrm{~mm}$. Les températures minimales et maximales

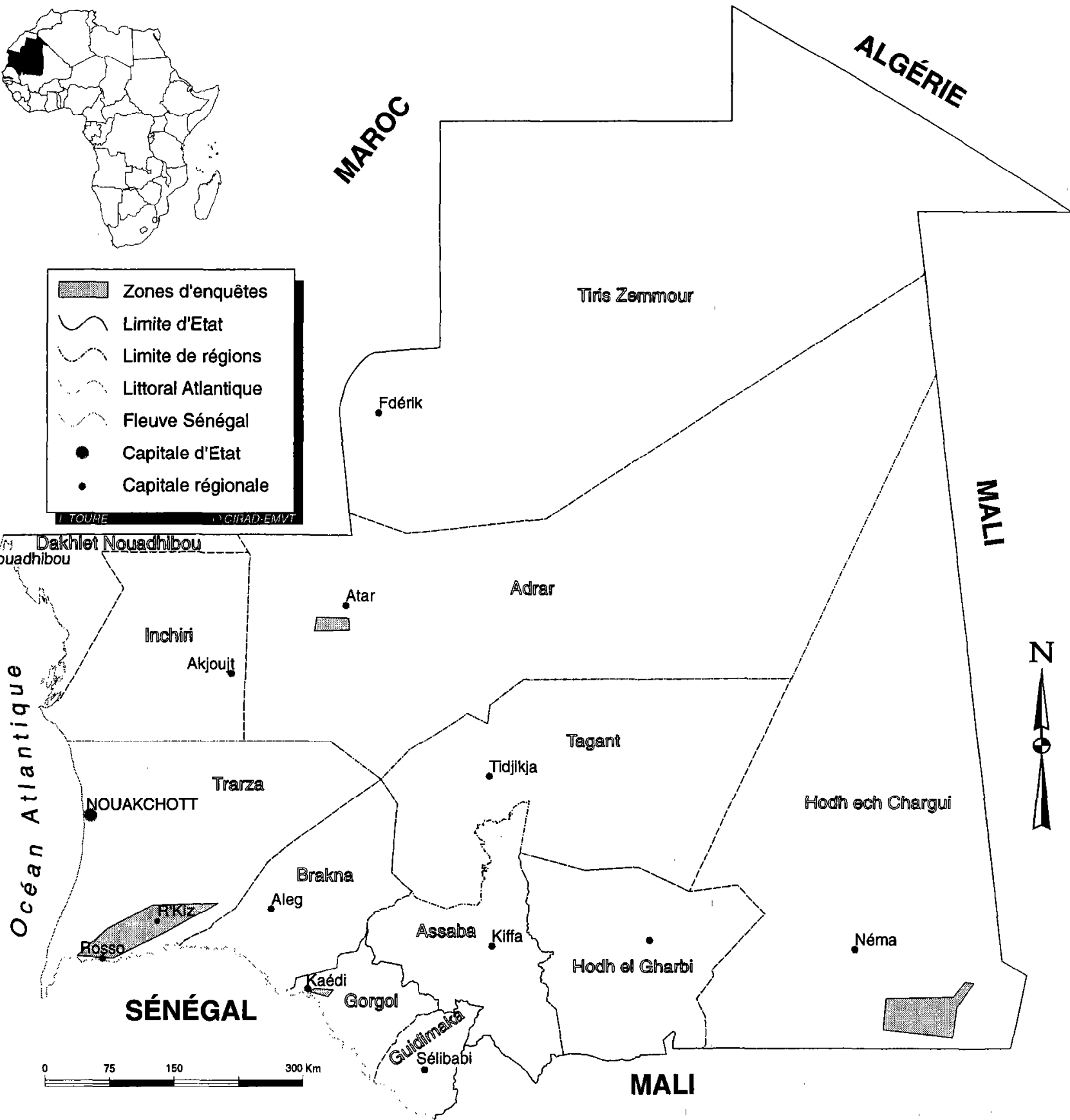

Figure 1 : origine des dromadaires enquêtés. 
moyennes sont de $24,3^{\circ} \mathrm{C}$ et $36,7^{\circ} \mathrm{C}$. L'humidité relative minimale et maximale moyenne est de 27 p. 100 et 50 p. 100. La végétation est composée de graminées à cycle court et de ligneux clairsemés (Acacia, Boscia senegalensis, Bauhinia rufescens, B. aegyptiaca, etc.)

\section{Hodh El Chargui}

La pluviométrie annuelle est comprise entre 250 et $350 \mathrm{~mm}$. Les températures moyennes minimales et maximales sont de $24,0^{\circ} \mathrm{C}$ et $37,3^{\circ} \mathrm{C}$. La région est très riche en pâturages avec des graminées pérennes (Aristida pungens, Panicum turgidum, etc.) et annuelles (Cenchrus biflorus, etc.). C'est une région d'élevage par excellence mais d'importantes ressources pastorales ne sont pas exploitées par manque de points d'eau pour abreuver les animaux.

\section{Adrar}

C'est une région désertique, avec d'importantes oasis. La pluviométrie moyenne de 1970 à 1995 était de $68 \mathrm{~mm}$. Les températures minimales et maximales sont de $21,2^{\circ} \mathrm{C}$ et $35,6^{\circ} \mathrm{C}$, mais de nombreuses données mensuelles des températures maximales manquent. L'humidité relative minimale et maximale moyenne est de 15 p. 100 et 42 p. 100

\section{Echantillonnage}

Au total 2078 dromadaires (tableau I) ont été prélevés au hasard des rencontres et du bon vouloir de l'éleveur ou du berger, contre des dons en produits vétérinaires ou alimentaires. Ils ont été regroupés en cinq classes d'âge ( $<1 ; 1-2 ; 2-5 ; 5-10 ;>10$ ans) et provenaient de 84 troupeaux. Trente-trois pour-cent des animaux étaient âgés de moins de 5 ans, le reste, 67 p. 100, était donc des adultes. Les mâles représentaient le quart de l'effectif.

Le Trarza est une région proche de Nouakchott et d'accès facile durant toute l'année. C'est pour cela que la majorité des prélèvements provenaient de cette région.

\section{Technique de mise en évidence de l'infection}

\section{Frottis sanguins}

Le sang était prélevé au niveau de la jugulaire dans des tubes contenant de l'EDTA. Les frottis sanguins, réalisés sur place, étaient colorés grâce à un kit de coloration rapide (RAL $\left.555^{\circledR}\right)$. L'examen des frottis était fait au CNERV. Les lames étaient observées au grossissement 400. Sur chaque lame, 50 champs microscopiques étaient examinés. Pour des raisons techniques (défaut de coloration, lames colorées brisées, etc.), ou parce que les prises de sang sur anticoagulant n'avaient pas été faites, la lecture des frottis n'a été réalisée que sur 1880 animaux.

\section{Sérologie}

Le sang était recueilli sur des tubes secs avec un activateur de la coagulation pour la récolte des sérums qui ont été testés selon trois techniques :

\section{- CATT/T. evansi (card agglutination test for trypanosomosis)}

Ce test d'agglutination rapide (5-7 min) est distribué sous forme de kit. Il a été mis au point conjointement par l'Université libre de Bruxelles (VUB) et l'Institut de médecine tropicale (IMT) d'Anvers. L'antigène est obtenu à partir d'un clone de trypanosomes sanguicoles, VAT RoTat $1 / 2$ dérivé d'un stock de T. evansi, isolé d'un buffle, en 1982 en Indonésie (2). Les sérums sont testés à la dilution 1/4. Les résultats sont lus selon un score allant de ,,- \pm+ à +++ .

- IFI (immunofluorescence indirecte)

Le stock de $T$. evansi utilisé pour la confection des antigènes a été isolé d'une chamelle en juillet 1990 dans la région du Trarza. Après des passages chez la souris, des stabilats ont été faits et conservés avec du diméthylsulfoxyde dans de l'azote liquide à partir de juillet 1991 au centre ORSTOM de Montpellier. Le 26 novembre 1993, un stabilat a été inoculé à Nouakchott à des souris. Celles-ci ont été sacrifiées au pic de la parasitémie (5-9 jours après l'infection). Au sang recueilli était ajouté du citrate de sodium à $10 \mathrm{p}$. 100. Une goutte de ce sang très parasité était déposée puis étalée sur des lames pour IFI (BioMérieux, Bleu Epoxy). Les lames d'antigènes ainsi confectionnées étaient séchées au ventilateur pendant plus de $30 \mathrm{~min}$, puis conservées à $-20^{\circ} \mathrm{C}$ jusqu'à leur emploi.

Le conjugué était un antisérum polyclonal anti- $\operatorname{IgG}$ de dromadaire produit sur lapin par le CIRAD-EMVT et conservé sous forme lyophilisée. Cet anti-IgG était alors couplé avec la fluorescéine (9). Le conjugué était ensuite conservé à $-20^{\circ} \mathrm{C}$. A l'aide du sérum d'un chamelon infecté pour la circonstance et celui d'un chamelon non infecté, différentes dilutions de ces sérums (1/20 au 1/1 280) et du conjugué $(1 / 20$ à $1 / 1000)$ ont été faites. Jusqu'à la dilution du conjugué au 1/80, la fluorescence était nette.

Les sérums à tester ont été dilués de 1/20 à 1/320. Le seuil de positivité retenu était de 1/80.

\section{- ELISA (enzyme-linked immunosorbent assay)}

L'anticorps monoclonal et le conjugué ont été produits par le Centre for tropical veterinary medicine d'Edimbourg (19). La dilution du monoclonal (MAB/IgG, $1 \mathrm{mg} / \mathrm{ml}$ ) était au $1 / 400$, celle du conjugué (MAB/HPO) au 1/5 000 et celle des sérums testés en double au 1/2. Les plaques utilisées étaient des plaques Immulon 2 à fond plat (Dynatec $®)$. Elles étaient gardées à $4^{\circ} \mathrm{C}$ jusqu'à leur emploi. Le substrat employé était le tétraméthylbenzidine dihydrochloride. La lecture était faite à $450 \mathrm{~nm}$ de longueur d'onde grâce

Tableau I

Composition des groupes d'animaux prélevés

\begin{tabular}{|c|c|c|c|c|c|c|c|c|}
\hline Région & Mâle & Femelle & $<1$ an & 1-2 ans & 2-5 ans & $5-10$ ans & $>10$ ans & Total \\
\hline Trarza Sud & 278 & 959 & 109 & 70 & 235 & 638 & 185 & 1237 \\
\hline Trarza Nord & 46 & 237 & 24 & 19 & 53 & 113 & 74 & 283 \\
\hline Trarza & 324 & 1196 & 133 & 89 & 288 & 751 & 259 & 1520 \\
\hline Gorgol & 68 & 142 & 0 & 2 & 59 & 89 & 60 & 210 \\
\hline Adrar & 28 & 53 & 5 & 3 & 4 & 22 & 47 & 81 \\
\hline Hodh Chargui & 109 & 158 & 31 & 23 & 56 & 122 & 35 & 267 \\
\hline Total & 529 & 1549 & 169 & 117 & 407 & 984 & 401 & 2078 \\
\hline
\end{tabular}


à un lecteur ELISA LP 400 (Diagnostic Pasteur). Le seuil de positivité retenu était une densité optique (DO) égale au moins à cinq fois la DO du témoin négatif.

Tout sérum qui donnait une réaction douteuse, ou des résultats différents selon les techniques utilisées, était testé deux fois.

L'examen parasitologique est de faible sensibilité par rapport aux tests sérologiques. Le résultat de chaque examen de frottis sanguin a été comparé à celui du sérum correspondant, pour établir lequel des tests faisait le mieux ressortir les vrais des faux positifs. La sensibilité et la spécificité chiffrées ont été déterminées selon la formule suivante :

Sensibilité observée $=$ p. 100 de séropositifs chez les animaux trypanosomés (frottis + )

Spécificité observée $=$ p. 100 de séronégatifs $($ ou \pm$)$ chez les animaux considérés comme non trypanosomés (frottis -)

\section{Analyse statistique}

Les résultats obtenus, ainsi que les facteurs de variation, ont été étudiés en leur appliquant des tests de comparaison (Chi 2) à l'aide du logiciel EPI INFO.

\section{RESULTATS}

\section{Prévalence totale}

\section{Recherche parasitologique}

Sur les 1880 dromadaires examinés en parasitologie, la prévalence était de 1,4 p. 100 (tableaux II). Les parasites observés avaient une morphologie caractéristique de T. evansi (groupe bru$c e i)$. Les parasitémies variaient de quelques trypanosomes par lame à plus de 20 par champ microscopique.

\section{Tests sérologiques}

Avec le CATT, le taux de séropositivité totale était de 16,5 p. 100. Les sérums de 6,0 p. 100 des animaux donnaient une faible agglutination, c'est-à-dire étaient douteux. Avec l'IFI, la séroprévalence était plus importante $(24,3$ p. 100$)$ avec 15,7 p. 100 de cas douteux. Quant à l'ELISA, le taux de positivité était de 14, 2 p. 100 avec 4,3 p. 100 de résultats douteux (tableaux II).

\section{Corrélation entre les quatre tests}

Pour des raisons évoquées plus haut, seuls 1880 dromadaires ont été examinés à la fois en parasitologie et en sérologie. En re-

Tableau II

Prévalence de l'infection (\%)

\begin{tabular}{|c|c|c|c|c|c|c|c|c|c|c|c|}
\hline \multirow[t]{2}{*}{ Région } & \multicolumn{2}{|c|}{ Frottis } & \multicolumn{3}{|c|}{ CATT } & \multicolumn{3}{|c|}{ IFI } & \multicolumn{3}{|c|}{ ELISA } \\
\hline & testés & + & testés & + & \pm & testés & + & \pm & testés & + & \pm \\
\hline Trarza & 1322 & $\begin{array}{r}24 \\
(1,8)\end{array}$ & 1520 & $\begin{array}{r}278 \\
(18,3)\end{array}$ & $\begin{array}{c}102 \\
(6,7)\end{array}$ & 1520 & $\begin{array}{r}439 \\
(29,0)\end{array}$ & $\begin{array}{r}252 \\
(16,6)\end{array}$ & 1520 & $\begin{array}{r}246 \\
(16,2)\end{array}$ & $\begin{array}{r}75 \\
(5,0)\end{array}$ \\
\hline Gorgol & 210 & $\begin{array}{r}2 \\
(0,9)\end{array}$ & 210 & $\begin{array}{r}34 \\
(16,2)\end{array}$ & $\begin{array}{r}14 \\
(6,6)\end{array}$ & 210 & $\begin{array}{r}34 \\
(16,2)\end{array}$ & $\begin{array}{r}19 \\
(9,0)\end{array}$ & 210 & $\begin{array}{r}22 \\
(10,5)\end{array}$ & $\begin{array}{r}7 \\
(3,3)\end{array}$ \\
\hline H. Chargui & 267 & 0 & 267 & $\begin{array}{r}25 \\
(9,4)\end{array}$ & $\begin{array}{r}6 \\
(2,2)\end{array}$ & 267 & $\begin{array}{r}27 \\
(10,1)\end{array}$ & $\begin{array}{r}28 \\
(10,5)\end{array}$ & 267 & $\begin{array}{r}16 \\
(6,0)\end{array}$ & $\begin{array}{r}4 \\
(1,5)\end{array}$ \\
\hline Adrar & 81 & 0 & 81 & $\begin{array}{r}6 \\
(7,4)\end{array}$ & $\begin{array}{r}4 \\
(5,0)\end{array}$ & 81 & $\begin{array}{r}5 \\
(6,2)\end{array}$ & $\begin{array}{r}27 \\
(33,3)\end{array}$ & 81 & $\begin{array}{r}11 \\
(13,6)\end{array}$ & $\begin{array}{r}4 \\
(4,5)\end{array}$ \\
\hline Total & 1880 & $\begin{array}{r}26 \\
(1,4)\end{array}$ & 2078 & $\begin{array}{r}343 \\
(16,5)\end{array}$ & $\begin{array}{r}126 \\
(6,0)\end{array}$ & 2078 & $\begin{array}{r}505 \\
(24,3)\end{array}$ & $\begin{array}{r}326 \\
(15,7)\end{array}$ & 2078 & $\begin{array}{r}295 \\
(14,2)\end{array}$ & $\begin{array}{r}90 \\
(4,3)\end{array}$ \\
\hline
\end{tabular}

Tableau III

Corrélation entre les différents tests

\begin{tabular}{|c|c|c|c|c|c|c|c|c|c|c|c|}
\hline & $F+$ & $\mathbf{F}-$ & CATT + & CATT \pm & CATT - & IFI + & IFI \pm & IFI - & ELISA + & EISA : & ELISA - \\
\hline Frottis + & 26 & & 21 & 3 & 2 & 24 & 2 & 0 & 13 & 0 & 13 \\
\hline Frottis - & & 1854 & 301 & 112 & 1441 & 454 & 308 & 1092 & 258 & 84 & 1512 \\
\hline CATT + & 21 & 301 & 343 & & & 245 & 55 & 43 & 125 & 11 & 207 \\
\hline CATT \pm & 3 & 112 & & 126 & & 54 & 33 & 39 & 28 & 7 & 91 \\
\hline CATT - & 2 & 1441 & & & 1609 & 206 & 238 & 1165 & 142 & 72 & 1395 \\
\hline IFI + & 24 & 454 & 245 & 54 & 206 & 505 & & & 162 & 20 & 323 \\
\hline IF I士 & 2 & 308 & 55 & 33 & 238 & & 326 & & 20 & 48 & 258 \\
\hline IFI - & 0 & 1092 & 43 & 39 & 1165 & & & 1247 & 113 & 22 & 1112 \\
\hline ELISA + & 13 & 258 & 125 & 28 & 142 & 162 & 20 & 113 & 295 & & \\
\hline ELISA \pm & 0 & 84 & 11 & 7 & 72 & 20 & 48 & 22 & & 90 & \\
\hline ELISA - & 13 & 1512 & 207 & 91 & 1395 & 323 & 258 & 1112 & & & 1693 \\
\hline
\end{tabular}


vanche, tous les animaux prélevés (2 078) ont été testés sérologiquement par les trois techniques (tableau III). La sensibilité et la spécificité observées étaient respectivement de 80,76 p. 100 et de 83,8 p. 100 pour le CATT, de 92,30 p. 100 et de 75,5 p. 100 pour l'IFI et de 50,0 p. 100 et 86,1 p. 100 pour l'ELISA.

Il existe des différences significatives entre ces tests : entre IFICATT et IFI-ELISA $\left(p<10^{-4}\right)$ et entre CATT-ELISA $(p<0,1)$. Entre les tests sérologiques (individuels ou combinés) et l'examen parasitologique, les différences étaient très significatives $\left(\mathrm{p}<10^{-3}\right.$ à $\left.10^{-5}\right)$.

\section{Facteurs de variation}

\section{Origine géographique des troupeaux}

L'infection variait en fonction de l'origine géographique des animaux (figure 2). D'après l'examen parasitologique, le CATT et l'IFI, les régions les plus infectées étaient le Trarza et le Gorgol. D'après l'ELISA, les régions les plus infectées étaient le Trarza, l'Adrar et le Gorgol. Le Trarza apparaît donc comme une région où l'infection était prédominante.

L'analyse statistique de ces régions comparées deux à deux montre que :

- avec le CATT, il existe des différences significatives entre Trarza ct Hodh El Chargui ( $p<0,001$ ), entre Trarza et Adrar $(p<0,01)$ et entre Gorgol et Hodh El Chargui $(p<0,1)$;

- avec l'IFI, il existe des différences très significatives entre Trarza et Gorgol $\left(\mathrm{p}<10^{-4}\right)$, entre Trarza et Hodh El Chargui $\left(\mathrm{p}<10^{-7}\right)$ et entre Trarza et Adrar $(p<0,001)$;

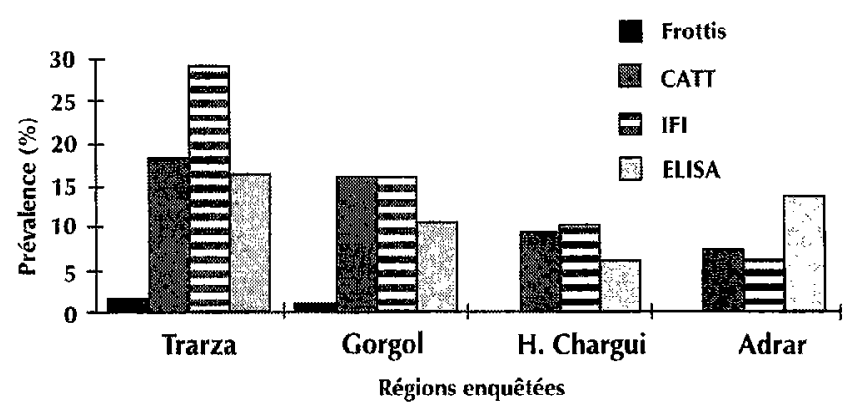

Figure 2 : prévalence de l'infection selon des régions et les tests de diagnostic.
- avec l'ELISA, on note des différences significatives entre Trarza et Gorgol ( $p<0,001)$, Trarza et Hodh El Chargui $\left(p<10^{-7}\right)$, entre Gorgol et Hodh El Chargui ( $p<0,1)$, entre Gorgol et Adrar ( $p<0,5)$.

\section{Stratégie de conduite de troupeau au sein d'une même région}

L'analyse statistique du tableau IV montre des différences d'infection très significatives entre les deux stratégies de conduite d'élevage ( $\mathrm{p}<0,00001)$.

Les animaux du sud étaient de loin plus fréquemment infectés que ceux du nord. Lors d'enquêtes entomologiques par les auteurs, c'est autour du Lac de R'kiz (sud du Trarza) qu'ils ont capturé de nombreux tabanides, principalement Atylotus agrestis, Tabanus taeniola et $T$. sufis, et des stomoxes, Haematobia minuta et $H$. irritans. Aucun de ces insectes n'a été capturé dans le nord de cette région.

\section{Age}

L'infection concernait tous les âges mais davantage les dromadaircs âgćs de 5-10 ans. Quel que soit le test utilisé, la courbe de la séroconversion avait le même aspect (figure 3).

L'analyse statistique a mis en évidence des différences très significatives entre les animaux de la classe des 5-10 ans et ceux des autres classes $(\mathrm{p}<0,001$ à 0,0001$)$. Le regroupement des animaux en jeunes ( $<5$ ans) et en adultes ( $>5$ ans) a également mis en évidence des différences très significatives entre ces deux groupes $\left(\mathrm{p}<10^{-5}\right)$.

\section{Sexe}

Avec les tests sérologiques combinés ou individuels, il existait des différences significatives entre les deux sexes, mais pas toujours dans le même sens $(p<0,05$ à $p<0,01)$. En revanche, avec l'examen parasitologique, il n'y avait pas de différence d'infection selon le sexe (tableau V).

\section{Tableau IV}

Prévalence selon la conduite de troupeau

\begin{tabular}{|c|c|c|c|c|c|c|c|c|}
\hline \multirow[b]{2}{*}{ Conduite délevage } & \multicolumn{2}{|c|}{ Frottis } & \multicolumn{2}{|c|}{ CATT } & \multicolumn{2}{|r|}{ IFI } & \multicolumn{2}{|c|}{ ELISA } \\
\hline & testes & $+(\%)$ & testés & $+(\%)$ & testés & $+(\%)$ & testés & $+(\%)$ \\
\hline Strategle sud & 1039 & $26(2,5)$ & 1237 & $271(21,9)$ & 1237 & $392(31,7)$ & 1237 & $226(18,3)$ \\
\hline Stratégie Nord: & 283 & $\mathbf{0}$ & 283 & $7(2,5)$ & 283 & $47(16,6)$ & 283 & $20(7,0)$ \\
\hline
\end{tabular}


Tableau V

Influence du sexe

\begin{tabular}{|c|c|c|c|c|c|c|c|c|}
\hline \multirow[b]{2}{*}{ Sexe } & \multicolumn{2}{|c|}{ Frottis } & \multicolumn{2}{|c|}{ CATT } & \multicolumn{2}{|c|}{ IFI } & \multicolumn{2}{|c|}{ ELISA } \\
\hline & testés & $+(\%)$ & testés & $+(\%)$ & testés & $+(\%)$ & testés & $+(\%)$ \\
\hline Mâles & 479 & $8(1,7)$ & 529 & $71(13,4)$ & 529 & $151(28,5)$ & 529 & $68(12,8)$ \\
\hline Femelles & 1401 & $18(1,3)$ & 1549 & $272(17,5)$ & 1549 & $354(22,8)$ & 1549 & $227(14,6)$ \\
\hline Total & 1880 & $26(1,4)$ & 2078 & $343(16,5)$ & 2078 & $505(24,3)$ & 2078 & $295(14,2)$ \\
\hline
\end{tabular}

\section{DISCUSSIO N}

Dans cette étude, deux aspects doivent être considérés : les techniques de diagnostic utilisées et les facteurs de variation de l'épidémiologie.

\section{Techniques de diagnostic}

Les résultats par examen parasitologique trouvés dans cette enquête étaient inférieurs à ceux de Christy, 5,4 p. 100 (5) et de Jacquiet et coll., 7,3 p. 100 (10). Le premier n'a examiné que des animaux douteux et le second a effectué une enquête limitée, alors que le présent travail concernait un effectif très important.

Les tests sérologiques, en particulier l'IFI, ont fait apparaître un taux d'infection plus élevé que l'examen parasitologique $(3,12)$ Le manque de sensibilité souvent attribué à la méthode de détection directe serait peut-être la cause principale de cette énorme différence entre les résultats parasitologiques et sérologiques. Ce taux d'infection parasitologique aurait probablement été plus élevé si la technique de centrifugation hématocrite avait été utilisée $(4,26)$ ou l'inoculation à des souris. Il est difficile d'appliquer ces deux méthodes sur le terrain en Mauritanie. Cependant, les résultats présentés ici sont proches de ceux de Diall et coll. dont l'enquête a révélé une séroprévalence de 30,60 p. 100 contre une prévalence parasitologique de 5,85 p. 100 au Mali (7).

D'après les pourcentages de co-positivité, les corrélations entre les différents tests sérologiques étaient les suivantes :

- 71,4 p. 100 des cas positifs au CATT l'étaient à l'IFI ;

- 48,5 p. 100 des cas positifs à l'IFI l'étaient au CATT ;

- 42,3 p. 100 des cas positifs au CATT l'étaient à l'ELISA ;

- 36,4 p. 100 des cas positifs à l'ELISA l'étaient au CATT ;

- 54,9 p. 100 des cas positifs à l'IFI l'étaient à l'ELISA ;

- 32,0 p. 100 des cas positifs à l'ELISA l'étaient à l'IFI.

Les résultats de l'ELISA paraissent décevants si l'on considère ceux d'autres auteurs $(15,17,18,19)$. Ces auteurs soulignent également son manque de sensibilité par rapport à des tests parasitologiques plus sensibles que celui utilisé dans cette étude $(6,15,25)$. Au Kenya, un dromadaire mort en phase aiguë d'infection à $T$. evansi était négatif à l'ELISA-Ag (24).

Dans cette étude, l'IFI s'est révélé comme le test le plus sensible mais le moins spécifique. Les cas douteux étaient considérables par rapport à ceux révélés par le CATT et l'ELISA. La sensibilité et la spécificité étaient satisfaisantes avec le CATT qui est, par ailleurs, un test facile à effectuer sur le terrain. Diall et coll. ont pu faire les mêmes observations lors de l'évaluation de ce test au Mali (7).

\section{Facteurs de variation de l'épidémiologie}

\section{Région et stratégie de conduite d'élevage}

La région est un important facteur de variation. L'infection à $T$. evansi a été observée dans toutes les régions mais surtout dans le Trarza, en particulier dans le sud de cette région, riche en cours d'eau. Il semblerait ainsi qu'une corrélation existe entre la prévalence de l'infection et la présence d'arbres et d'arbustes près de ces cours d'eau. En effet, les premiers éléments de cette enquête sur l'entomofaune menée dans le sud du Trarza ont permis d'identifier trois espèces de tabanides et deux espèces de stomoxe susceptibles de transmettre $T$. evansi. Ces insectes, présents toute l'année dans la zone, étaient très abondants en fin d'hivernage (octobre-novembre). Or, en Adrar, les éleveurs connaissaient peu ces insectes. Au Hodh El Chargui, seuls des hippobosques ont été récoltés à l'aide de filets à main et leur importance épidémiologique a paru moindre que celle des tabanides et des stomoxes.

La stratégie de conduite du troupeau est aussi un facteur non négligeable. Les résultats de l'examen parasitologique, du CATT et de l'ELISA, principalement, ont révélé que les dromadaires transhumant vers le sud étaient les plus affectés.

\section{Effet troupeau}

Dans une même région, le taux d'infection variait d'un troupeau à un autre. Dans certains troupeaux les séroprévalences étaient supérieures à 60 p. 100, alors que dans d'autres elles étaient nulles. Ceci variait selon la situation des éleveurs, l'utilisation plus ou moins grande de trypanocides étant favorisée par la facilité pour s'en procurer ou par la proximité des services vétérinaires. Des bergers ont fréquemment demandé conseil aux auteurs à propos des trypanocides remis par le propriétaire des animaux (souvent cadre supérieur).

\section{Age des animaux}

Des trypanosomes ont été observés dans le sang périphérique de jeunes animaux âgés de 8-10 mois. Ceci remet en cause le constat d'absence d'infection chez le jeune chamelon signalée par certains auteurs $(6,10)$.

La séroprévalence était la plus élevée chez les animaux âgés de 510 ans pour décroître ensuite. Si l'accroissement de la séroprévalence de l'infection à partir de 2 ans jusqu'à 10 ans peut s'expliquer par l'augmentation de la probabilité de rencontre avec des insectes porteurs de T. evansi, il est difficile en revanche de se prononcer sur la diminution constatée chez les animaux âgés de 0-2 ans et surtout chez les plus de 10 ans. Pour les plus âgés, cela pourrait s'expliquer par une immunisation progressive contre des trypanosomes, ou par une baisse de la longévité lorsqu'ils ont été infectés par des trypanosomes, ou par leur envoi rapide à la boucherie par des éleveurs avertis. En effet, dans une autre étude menée à l'abattoir de Nouakchott, les auteurs ont trouvé des prévalences de l'ordre de 30 p. 100. 


\section{CONCLUSION}

La trypanosomose cameline à $T$. evansi est bien présente en Mauritanie. Elle se répartit de façon variable selon les régions et la stratégie de conduite du troupeau. L'importance de la maladie serait probablement liée à la présence d'eaux de surface favorisant les couverts végétaux, fréquentés par les animaux, offrant ainsi des conditions favorables au développement d'insectes vecteurs de ce parasite.

La prévalence parasitologique est très faible par rapport à la séroprévalence. Celle-ci est plus forte avec l'IFI qu'avec le CATT et l'ELISA. Elle atteint un maximum chez les animaux âgés de 10 ans pour décroître ensuite. La sensibilité est meilleure avec l'IFI et la spécificité est excellente avec l'ELISA. Avec le CATT, la sensibilité et la spécificité sont satisfaisantes.

\section{Remerciements}

Ce travail a reçu l'appui financier de l'Unité de coordination d'élevage des camelins (UCEC) du CIRAD-EMVT, de la Fondation internationale pour la science (IFS) et de l'AUPELF. Nous remercions également Cheikh Lebchir et Alioune M'Rezig pour leur aide très précieuse.

\section{BIBLIO GRAPHIE}

1. BAILEY J.W., SMITH D.H., 1992. The use of acridine orange QBC technique in the diagnosis of African trypanosomosis. Trans. R. Soc. trop. Med. Hyg., 86: 630.

2. BAJYANA SONGA E., HAMERS R.,1988. A card agglutination test (CATT) for veterinary use based on an early VAT RoTat $1 / 2$ of T. evansi. Ann. Soc. belge Med. trop., 68: 233-240.

3. BOCQUENTIN R., VERY P., DUVALLET G., 1990. Cinétique des anticorps après traitement trypanocide chez les bovins infectés expérimentalement ou naturellement. Intérêt épidémiologique. Revue Elev. Méd. vét. Pays trop., 43 : 479-483.

4. CAMUS E., 1983. Diagnostic de la trypanosomose bovine sur le terrain par la méthode de centrifugation hématocrite. Revue sci. tech. O ff. int. Epiz., 2 : 751-769.

5. CHRISTY P., 1987. Enquête sur les trypanosomes du dromadaire en Mauritanie. Nouakchott, Mauritanie, CN ERV, p. 5-9. (Rapport d'activité)

6. DIALL O., BAJYANA SONGO E., MAGNUS E., KOUYATE B., DIALLO B., VAN MEIRVENNE N., HAMERS R., 1994. Evaluation d'un test sérologique d'agglutination directe sur carte dans le diagnostic de la trypanosomose cameline à T. evansi. Revue sci. tech. O ff. int. Epiz., 13 : 793-800.

7. DIALL O., BO COUM Z., DIARRA B., SANOGO Y., COU LIBALY Z., WAIGALO Y., 1993. Epidémiologie de la trypanosomose à T. evansi chez le dromadaire au Mali : résultats d'enquêtes parasitologiques et cliniques. Revue Elev. Méd. vét. Pays trop., 46 : 455-461.

8. FAO, 1993. Développement de l'élevage en République islamique de Mauritanie. Rome, Italie, FAO, 168 p. (Rapport de N ouakchott, 1993)
9. IN STITUT PASTEUR, 1973. Cours sur les techniques de l'immunofluorescence appliquées aux problèmes d'intérêt vétérinaire. Paris, France, Institut Pasteur, p. 9-19.

10. JACQUIET P., DIA M.L., CHEIKH D., THIAM A., 1994. La trypanosomose cameline à Trypanosoma evansi (Steel 1885), Balbiani 1888, en République islamique de Mauritanie : Résultats d'enquêtes dans le Trarza. Revue Elev. Méd. vét. Pays trop., 47 : 59-62.

11. LEVINE R.A., WARDLAW S.C., PATTON C.L., 1989. Detection of haematoparasites using quantitative buffy coat analysis tubes. Parasitol. Today, 5: 132-133.

12. LUCKINS A.G., 1977. Detection of antibody in trypanosomeinfected cattle by means of a microplate enzyme-linked immunosorbent assay. Trop. Anim. Health Prod., 9: 52-63.

13. LU CKIN S A.G., MEHLITZ, 1980. Evaluation of an indirect fluorescent antibody test, enzyme-linked immunosorbent assay and quantification of immunoglobulins in the diagnosis of bovine trypanosomosis. Trop. Anim. Health Prod., 10: 149-159.

14. MAHMOUD M.M., GRAY A.R., 1980. Trypanosomosis due to $T$. evansi (Steel, 1885) Balbiani, 1888. A review of recent research. Trop. Anim. Health Prod., 12: 35-47.

15. MASAKE R.A., MOLOO S.K., NANTULYA V.M., MINJA S.H., MAKAU J.M., NJUGUNA J.T., 1995. Comparative sensitivity of antigen detection enzyme immunosorbent assay and microhematocrit centrifugation technique in the diagnosis of Trypanosoma brucei infections in cattle. Vet. Parasitol., 56: 37-46.

16. NANTULYA V.M., 1989. Surratex: A simple latex agglutination antigen test for diagnosis of Trypanosoma evansi infections (Surra). Trop. Med. Parasitol., 45: 9-12.

17. NANTULYA V.M., LINDQVIST K.J., DIALL O., OLAHO M.W. 1989. Two simple antigen-detection immunoassays for the diagnosis of Trypanosoma evansi infections in the dromedary camel (Camelus dromedarius). Trop. Med. Parasitol., 40: 415-418.

18. OLAHO M.W., MUNYUA W.K., MUTUGI M.W., NJOGU A.R., 1993. Comparison of antibody-and antigen-detection enzyme immunoassays for the diagnosis of Trypanosoma evansi infections in camels. Vet. Parasitol., 45: 231-240.

19. RAE P.F., LUCKINS A.G., 1984. Detection of circulating trypanosomal antigens by enzyme immunoassays. Ann. trop. Med. Parasitol., 78: 587-596.

20. RAE P.F., LUCKINS A.G., 1992. Problems in the diagnosis of cameline trypanosomosis. In: Proc. first int. camel conf., Dubai, U nited Arab Emirates, February 2-6, 1992, p. 29-31.

21. SINGH V., CHHABRA M.B., 1993. Counter immuno-electrophoresis for rapid detection of circulating antigens of $\mathrm{T}$. evansi. Indian $\mathrm{J}$. Anim. Sci., 63: 625-627.

22. VENKATEJAN P., WAKELIN D., 1993. Elisas for parasitologists: or lies, damned lies and Elisas. Parasitol. Today, 9: 228-232.

23. VERMA B.B., GAUTAM O.P., 1977. Serological diagnosis of experimental bovine surra (Trypanosoma evansi infection). A comparison of passive haemagglutination, gel diffusion and indirect fluorescent antibody tests. Indian vet. J., 54: 809-813.

24. WAITUMBI J. N., NANTULYA V. M., 1993. A comparison of the antigen detection ELISA and parasite detection for diagnosis of Trypanosoma evansi infections in camels. Vet. Parasitol., 49: 159-178.

25. WAITHANJI E.M., NANTULYA V.M., MBIUKI S.M., 1983. Use of antigen capture tube enzyme-linked immunosorbent assay for the diagnosis of Trypanosoma evansi infections in dromedary camels (Camelus dromedarius). Revue sci. tech. O ff. int. Epiz., 12: 665-672.

26. Wo 0 P.T.K., 1970. The haematocrit centrifuge technique for the diagnosis of African trypanosomosis. Acta Trop., 27: 384-386.

Reçu le 3.1.97, accepté le 7.7.97 


\section{Summary}

Dia M.L., Van Meirvenne N., Magnus E., Luckins A.G ., Diop C., Thiam A., Jacquiet P., Hamers R. Evaluation of four diagnosis tests: blood smears, CATT, IFAT and ELISA-Ag detection in a study of the epidemiology of T. evansi camel trypanosomosis in Mauritania

A study was conducted on the epidemiology of camel trypanosomosis caused by T. evansi in M auritania using 2078 one-humped camels of different ages from four regions with different climates and ecology (Trarza, Gorgol, Adrar and Hodh El Chargui). The prevalence of the infection was determined by blood smear examinations and three serological tests, the card agglutination test for trypanosomosis (CATT), an indirect fluorescent antibody test (IFAT) and an enzyme linked immunosorbent assay (ELISA) for the detection of trypanosomal antigens. The overall parasitological prevalence of the infection was $1.4 \%$; seropositivity rates were $16.5 \%$ with CATT, $24.3 \%$ with IFAT and $14.1 \%$ using antigen-detection ELISA. Prevalence rates changed depending on the region, the herd, the age of the camels and herd management strategy. The study showed that camel trypanosomosis was widespread in Mauritania, especially in the wooded areas close to waterways used by animals.

Key words: Dromedary - Trypanosoma evansi - Agglutination tests - Immunofluorescence - ELISA - Agroclimatic region Livestock management - Epidemiology - Mauritania.

\section{Resumen}

Dia M.L., Van Meirvenne N ., Magnus E., Luckins A.G ., D iop C., Thiam A., Jacquiet P., Hamers R. Evaluación de cuatro tests diagnósticos: frotis sanguíneos, CATT, IFI y ELISA-Ag en el estudio de la epidemiología de la tripanosomosis de los camélidos por Tripanosoma evansi en Mauritania

Se llevó a cabo una encuesta sobre la epidemiología de la tripanosomosis en el camello por T. evansi en Mauritania, en una muestra de 2078 dromedarios de todas las edades, en quatro regiones (Trarza, Gorgol, Adrar, Hodh El Chargui) con características climáticas y ecológicas diferentes. La prevalencia de la infección se determinó mediante el examen de frotis sanguíneos y por tres tests serológicos, el test de aglutinación en carta para tripanosomosis (CATT), la inmunofluorescencia indirecta (IFI) para la detección de anticuerpos y el ensayo inmunoabsorbente ligado a enzimas (ELISA) para la detección de antígenos. La prevalencia global de la infección fue de $1,4 \%$ mediante el examen parasitológico. La seroprevalencia fue de $16,5 \%$ por el CATT, de $24,3 \%$ por el IFI y de $14,1 \%$ por el ELISA-Ag. La prevalencia varió según la región, la estrategia de prácticas de manejo de los productores, los hatos y la edad de los animales. Esta encuesta demuestra que la tripanosomosis en los camélidos está presente en Mauritania, sobretodo en las zonas boscosas, cerca de los lechos de agua frecuentados por los animales.

Palabras clave: D romedario - Trypanosoma evansi - Reacción de aglutinación - Inmunofluorescencia - ELISA - Region agroclimática - Manejo del ganado - Epidemología Mauritania. 